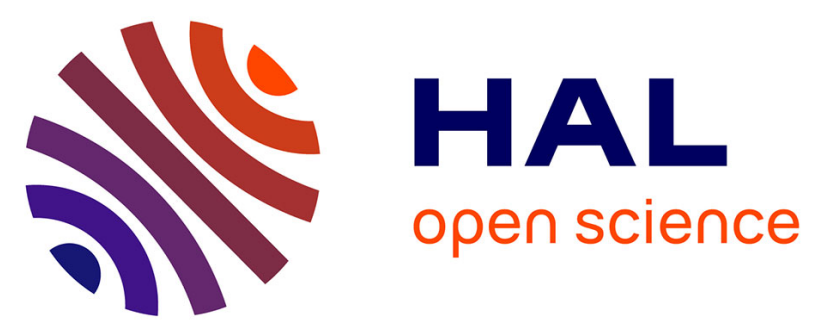

\title{
The tolerance of the human body to automobile collision impact - a systematic review of injury biomechanics research, 1990-2009.
}

Jason L. Forman, Francisco J. Lopez-Valdez, Sonia Duprey, Dipan Bose, Eduardo del Pozo de Dios, Damien Subit, Tim Gillispie, Jeff R. Crandall,

Maria Segui-Gomez

\section{To cite this version:}

Jason L. Forman, Francisco J. Lopez-Valdez, Sonia Duprey, Dipan Bose, Eduardo del Pozo de Dios, et al.. The tolerance of the human body to automobile collision impact - a systematic review of injury biomechanics research, 1990-2009.. Accident analysis and prevention, 2015, 80, pp.7-17. 10.1016/j.aap.2015.03.004 . hal-02488916

\section{HAL Id: hal-02488916 https://hal.science/hal-02488916}

Submitted on 24 Feb 2020

HAL is a multi-disciplinary open access archive for the deposit and dissemination of scientific research documents, whether they are published or not. The documents may come from teaching and research institutions in France or abroad, or from public or private research centers.
L'archive ouverte pluridisciplinaire HAL, est destinée au dépôt et à la diffusion de documents scientifiques de niveau recherche, publiés ou non, émanant des établissements d'enseignement et de recherche français ou étrangers, des laboratoires publics ou privés. 


\title{
The tolerance of the human body to automobile collision impact - a systematic review of injury biomechanics research, 1990-2009
}

\author{
Jason L. Forman ${ }^{\mathrm{a}, *}$, Francisco J. Lopez-Valdes ${ }^{\mathrm{b}}$, Sonia Duprey ${ }^{\mathrm{c}}$, Dipan Bose ${ }^{\mathrm{d}}$, \\ Eduardo del Pozo de Dios ${ }^{\mathrm{e}}$, Damien Subit ${ }^{\mathrm{f}}$, Tim Gillispie ${ }^{\mathrm{a}}$, Jeff R. Crandall ${ }^{\mathrm{a}}$, \\ Maria Segui-Gomez ${ }^{\mathrm{g}}$
}

\author{
a University of Virginia Center for Applied Biomechanics, 4040 Lewis and Clark Dr., Charlottesville, VA 22911, USA \\ b University of Zaragoza, Zaragoza, Spain \\ ' Laboratoire de Biomécanique et Mécanique des Chocs, IFSTTAR, Lyon, France \\ d Global Road Safety Facility, World Bank Group, Washington D.C., USA \\ e Applus IDIADA Group, Spain \\ ${ }^{\mathrm{f}}$ LBM - Institut de Biomécanique Humaine Georges Charpak, Arts et Métiers ParisTech, Paris, France \\ ${ }^{g}$ Director General for Traffic, Ministry of Interior, Spain
}

Keywords:

Automobiles

Biomechanics

Injuries

Prevention

Surveillance

Trauma

\begin{abstract}
A B S T R A C T
Road traffic injuries account for 1.3 million deaths per year world-wide. Mitigating both fatalities and injuries requires a detailed understanding of the tolerance of the human body to external load. To identify research priorities, it is necessary to periodically compare trends in injury tolerance research to the characteristics of injuries occurring in the field. This study sought to perform a systematic review on the last twenty years of experimental injury tolerance research, and to evaluate those results relative to available epidemiologic data. Four hundred and eight experimental injury tolerance studies from 1990-2009 were identified from a reference index of over 68,000 papers. Examined variables included the body regions, ages, and genders studied; and the experimental models used. Most (20\%) of the publications studied injury to the spine. There has also been a substantial volume of biomechanical research focused on upper and lower extremity injury, thoracic injury, and injury to the elderly - although these injury types still occur with regularity in the field. In contrast, information on pediatric injury and physiological injury (especially in the central nervous system) remains lacking. Given their frequency of injury in the field, future efforts should also include improving our understanding of tolerances and protection of vulnerable road users (e.g., motorcyclists, pedestrians).
\end{abstract}

\section{Introduction}

Automobile collisions are the most common source of severe unintentional injury worldwide (Chandran et al., 2010). Every year, more than 1.3 million people die and 50 million people are severely injured in road traffic crashes (Peden et al., 2004). It is projected that by 2020 , fatal and nonfatal road traffic injuries will increase by approximately 65 percent (Kopits and Cropper, 2003), and will be the sixth leading cause of death (Murray and Lopez, 1997) and the third leading cause of disability adjusted life years (DALYs) lost (Peden et al., 2004) world-wide. This projected increase in burden relates to an expansion of motorized transport, combined with shifts in road-user demographics to populations more susceptible to injury (e.g., pedestrians, the elderly). In response to this growing pandemic, the United Nations has declared 2011-2020 as the Decade for Action, with a goal of halving the number of world road traffic fatalities.

A critical component of the injury prevention effort is the understanding of injury tolerances. The human body can bear a certain amount of mechanical input - force, acceleration, compression, etc., - before a tissue failure or dysfunction occurs. This transition to a load that results in mechanical or functional tissue failure is known as the injury tolerance. In the automotive field, injury tolerance information informs engineers and 
designers on what body structures can be safely loaded, allows them to predict injury risk, dictates the design of vehicle safety features, and serves as the basis for regulations and assessment procedures for vehicle safety. Target scenarios and risk factors for intervention are often identified through epidemiology. When engineering solutions are indicated - for example, through improved vehicle restraints - injury biomechanics researchers work with policy makers, automobile manufacturers, and other stakeholders to identify strategies for intervention. Knowledge on injury tolerances provides insight into the causes of injuries in the field and provides performance targets for the evaluation of possible interventions prior to field implementation. Once implemented, the cycle renews with continuing epidemiology to observe the effectiveness of the interventions in reducing deaths or injuries, to identify opportunities for the further refinement of those intervention strategies, and to identify new research priorities. Injury tolerance information also allows the identification of differential intervention strategies based on specific road user characteristics, especially for at-risk populations (for example, the elderly (Kent et al., 2005a)).

It is pertinent to periodically review trends in injury tolerance research compared to injury frequencies, injury types, affected populations, and causation scenarios observed in the field. The last such review was performed by Viano et al. (1989) as a part of a treatise on the fundamentals of injury biomechanics. Several deficiencies in injury tolerance information were noted, including knowledge on functional injury to the central nervous system, structural spinal injury, functional injury to the heart and great vessels, lung injury, injury to the hollow abdominal organs, injury to the joints and long-bones of the extremities (other than the femur), and injury to the face and sensory organs. The only body structures for which "somewhat understood and verified" or "well known" injury tolerance information were available were the skull, the ribcage, the solid organs of the abdomen, and the femur. Children and adult females were identified as populations for which little information was available. There was also little mention of differential injury tolerances based on advanced age, body-type, or existing pathologies.

The goal of this study is to perform a systematic review of injury tolerance investigations in the 20 years following the review of Viano et al. (1989), and to compare trends therein to the road traffic injuries occurring in the field. To define the scope, this study focused on papers describing new injury tolerance information applicable to the motor-vehicle collision environment, derived from experimental studies with biological (not artificial or computational) models. A descriptive analysis was performed to study trends in the body regions, injury types, ages and genders studied, and the types of models used. Those results were then compared to the previous state of knowledge and to motor-vehicle injury trends observed in the epdidemiologic literature.

\section{Method}

\subsection{Literature database}

Papers were selected from a custom index of reference information (housed and maintained by the University of Virginia Center for Applied Biomechanics) for approximately 68,000 scholarly papers, reports, books, book chapters, and theses relating to injury biomechanics, biomedical engineering, automobile engineering, and automobile safety (referred to here as the Index). The Index spans from the year 1840 to the present, and is populated by monthly updates of 140 journals and conference proceedings for keywords related to injury biomechanics and automobile safety (e.g., 'traffic', 'biomechanics', 'safety', etc.). Index entries are stored in a searchable Access (Professional Edition 2003, Microsoft) database.

\subsection{Paper selection criteria}

This study targeted publications that included new experimental data describing injury tolerances with biological models. The inclusion criteria were as follows:

- Published between 1990 and 2009 (inclusive).

- Published in English.

- Included data recorded from experiments with biological models (e.g., human volunteers, cadavers, animals, or cell cultures).

- Included information on injuries or tissue failures that occurred during the experiments. This included either mechanical tissue failure (for example, breaking of an isolated bone segment or bone sample), or physiological injury (for example, mild traumatic brain injury in an animal model). Non-injury, living human, volunteer studies were included when they could be used for determining a lower-bound for the estimation of a tolerance for physiological injury. Non-injurious human cadaver studies were included only if they specifically sought to study injury tolerances, but happened to not cause injury in their experiments. Observational studies (studies observing real-life human exposures and resulting injuries in a non-laboratory setting) were included only if the observational subjects were instrumented or recorded to the extent that the mechanical inputs into the body could be reasonably deduced.

- Related to acute injury of mechanical origin in otherwise healthy tissue. This was not necessarily limited to papers specifically targeting the automotive environment because injury tolerance information from other fields (e.g., sports) can potentially be applied to the automotive environment. Papers relating to repetitive stress, injury in pathological tissue, injury in prosthetic interfaces, penetrating injury (e.g., gunshots and stabbing), blast injury, burns, and drowning were excluded.

- Included data not previously published. Review papers and papers describing amalgamations of previously-published data were excluded.

\subsection{Query method}

To facilitate categorization of papers by body region, the Index was queried with each of the body-region-specific search terms listed in Table 1. Queries were performed as an all-field search, returning entries based on search-term matches in the title, keywords, or abstract. Six reviewers were assigned one or two body regions each for which to perform queries and initial reviews. Once queried for each search term, the returned entries were reviewed to identify those possibly meeting the selection criteria. The resulting candidate papers were then obtained and reviewed in full to determine inclusion or exclusion in a provisional database.

\subsection{Variables}

Information to be extracted during the review process was operationalized in a dictionary that was used to develop a webbased extraction form used throughout the study. The reviewers were trained for consistency. The study leader reviewed all entries prior to closing the review process to check for repeat entries, missing values, and for consistency with the paper selection criteria. 
Table 1

Search terms used for all-field queries (title, abstract, keywords) of the reference index, organized by body region.

\begin{tabular}{ll}
\hline Body region & Search terms \\
\hline Head & Head; skull; brain; face \\
Spine and neck & Spine; neck; annulus; vertebra; disc; spinal cord \\
Thorax & Thorax; chest; rib; sternum; heart; lung; aorta; diaphragm \\
Abdomen & Abdomen; abdominal; liver; kidney; stomach; bowel; spleen; mesentery; intestine; bladder \\
Pelvis & Pelvis; pelvic; acetabulum \\
Upper extremities & Arm; upper extremity; hand; wrist; finger; clavicle; shoulder; scapula; humerus; radius; ulna; elbow \\
Lower extremities & Lower extremity; thigh; femur; knee; patella; tibia; fibula; leg; foot; ankle; hip \\
Other & Skin \\
\hline
\end{tabular}

Variables were defined to categorize the body regions and anatomical structures studied, the ages studied, the genders studied, the models used, and the specificity of the studies (i.e., were the results generally applicable or did they target a specific type of road user; Table 2).

The experimental models used were classified as either 'living human' (non-injurious volunteer tests or observational studies), 'human cadaver', 'animal', or 'human other' (in vitro tests either with living human cells or with post-delivery, extra-fetal, natal tissue such as the amnion or fetal membrane).

Ages were binned into three groups - age $<22$; age 23-65; and age $>65$. Age 22 was chosen for the transition from the youngest to the middle age group because that represents the approximate age of full skeletal maturity (Franklyn et al., 2007). In most cases, the ages covered by the publications were determined based on the ages of the test subjects used in the experiments. Multiple age groups were noted if a study used subjects spanning multiple age groups. For example, a study that performed experiments on cadaver tissue with an age range of 30-70 years would be counted in both the 23-65 year age group and the $>65$ age group. For animal studies, the age was categorized as 'general' (generally applicable) unless the study intended to target a specific age group. For example, a porcine study that specifically intended to investigate pediatric abdominal injury tolerance (Kent et al., 2008) was categorized in the $0-22$ age group.

Genders were categorized as male-focused, female-focused, or applicable to either. For studies involving humans or cadaver tissue the gender was categorized based on the characteristics of the subjects. Studies with only male subjects were categorized as 'male-focused'; studies with only female subjects were termed 'female-focused'. Studies that included both male and female subjects were categorized as 'general'. Animal studies were also categorized as 'general'.

The specificity of the studies was categorized either as 'general', 'car occupant', or 'pedestrian'. 'General' was used if the study provided information on general injury tolerances, not necessarily restricted to a particular injury causation scenario.

The selected papers were also categorized by the body region and anatomical structure studied. Those classifications were chosen based on an expansion of the taxonomy used in the injury

Table 2

Percent and 95\% confidence intervals (C.I.) for experimental injury tolerance investigations, 1990-2009 ( $n=408$ studies).

\begin{tabular}{|c|c|c|c|}
\hline & $\begin{array}{l}\text { Variable } \\
\text { Peer reviewed }\end{array}$ & $\begin{array}{l}\text { Percentage } \\
94.1\end{array}$ & $\begin{array}{l}\text { 95\% C.I. } \\
91.8,96.4\end{array}$ \\
\hline \multirow[t]{4}{*}{ Model } & Living human ${ }^{\mathrm{a}}$ & 6.9 & $4.4,9.3$ \\
\hline & Human cadaver & 74.5 & $70.3,78.7$ \\
\hline & Animal & 17.6 & $13.8,21.1$ \\
\hline & Other human ${ }^{\mathrm{b}}$ & 1.0 & $0.0,1.9$ \\
\hline \multirow[t]{4}{*}{ Gender } & Male & 12.7 & $9.5,16.0$ \\
\hline & Female & 5.6 & $3.4,7.9$ \\
\hline & Both or general $^{\mathrm{C}}$ & 65.7 & $61.1,70.3$ \\
\hline & Missing & 15.9 & $12.4,19.5$ \\
\hline \multirow[t]{5}{*}{ Age $(\text { years })^{d}$} & $0-22$ & 9.8 & $6.9,12.7$ \\
\hline & $23-65$ & 59.8 & $55.0,64.6$ \\
\hline & $>65$ & 59.6 & $54.8,64.3$ \\
\hline & General $^{\mathrm{e}}$ & 15.7 & $12.2,19.2$ \\
\hline & Missing & 10.3 & $7.3,13.2$ \\
\hline \multirow[t]{3}{*}{ Specificity } & General & 65.0 & $60.3,69.6$ \\
\hline & Car driver or occupant & 31.6 & $27.1,36.1$ \\
\hline & Pedestrian & 3.4 & $1.7,5.2$ \\
\hline \multirow[t]{9}{*}{ Body region } & Head & 15.7 & $12.2,19.2$ \\
\hline & Spine & 20.1 & $16.2,24.0$ \\
\hline & Thorax & 15.4 & $11.9,18.9$ \\
\hline & Abdomen & 5.9 & $3.6,8.2$ \\
\hline & Pelvis & 7.1 & $4.6,9.6$ \\
\hline & Upper extremities & 19.1 & $15.3,22.9$ \\
\hline & Lower extremities & 13.5 & $10.2,16.8$ \\
\hline & Sensory organs & 2.9 & $1.3,4.6$ \\
\hline & Neck (non-spinal) & 0.2 & $-0.2,0.7$ \\
\hline
\end{tabular}

a Non-injurious volunteer studies or human observational studies.

b Human cell cultures or post-delivery, extra-fetal, natal tissue.

c Includes living human and cadaver studies that used both male and female subjects, and animal studies.

d Some studies spanned multiple age categories.

e Animal studies not targeting a specific age range. 
Table 3

Percent distribution of body regions, sub-structures, models, and genders studied in experimental injury tolerance investigations, $1990-2009$ ( $n=408$ studies).

\begin{tabular}{|c|c|c|c|c|c|c|c|c|c|}
\hline \multirow[t]{2}{*}{ Body regions and sub-structures ${ }^{a}$} & \multicolumn{4}{|l|}{ Model } & \multicolumn{4}{|c|}{ Gender } & \multirow[t]{2}{*}{ Total } \\
\hline & Living human ${ }^{\mathrm{b}}$ & Human cadaver & Animal & Other human ${ }^{c}$ & Male & Female & Both or general $^{\mathrm{d}}$ & Missing & \\
\hline Head & 1.0 & 6.1 & 8.1 & 0.5 & 1.2 & 0.0 & 11.0 & 3.4 & 15.7 \\
\hline Skull & 0.0 & 5.1 & 0.7 & 0.0 & 0.2 & 0.0 & 2.2 & 3.4 & 5.9 \\
\hline Face & 0.0 & 1.0 & 0.0 & 0.0 & 0.0 & 0.0 & 1.0 & 0.0 & 1.0 \\
\hline Brain structure & 0.2 & 0.0 & 0.5 & 0.0 & 0.2 & 0.0 & 0.5 & 0.0 & 0.7 \\
\hline Brain function & 0.7 & 0.0 & 6.9 & 0.5 & 0.7 & 0.0 & 7.4 & 0.0 & 8.1 \\
\hline Spine & 4.7 & 11.8 & 3.7 & 0.0 & 2.9 & 1.0 & 13.7 & 2.5 & 20.1 \\
\hline Vertebrae & 0.0 & 2.5 & 0.7 & 0.0 & 0.2 & 0.2 & 2.0 & 0.7 & 3.2 \\
\hline Ligaments* & 0.0 & 1.2 & 0.5 & 0.0 & 0.7 & 0.0 & 1.0 & 0.0 & 1.7 \\
\hline Vertebrae and ligament structure* & 4.7 & 8.1 & 1.0 & 0.0 & 2.0 & 0.7 & 9.3 & 1.7 & 13.7 \\
\hline Spinal cord structure & 0.0 & 0.0 & 1.0 & 0.0 & 0.0 & 0.0 & 1.0 & 0.0 & 1.0 \\
\hline Spinal cord function & 0.0 & 0.0 & 0.5 & 0.0 & 0.0 & 0.0 & 0.5 & 0.0 & 0.5 \\
\hline Thorax & 0.5 & 13.2 & 1.7 & 0.0 & 3.4 & 0.5 & 10.5 & 1.0 & 15.4 \\
\hline Ribcage & 0.5 & 10.8 & 1.0 & 0.0 & 3.2 & 0.5 & 7.8 & 0.7 & 12.3 \\
\hline Heart and great vessel structure & 0.0 & 2.2 & 0.0 & 0.0 & 0.2 & 0.0 & 1.7 & 0.2 & 2.2 \\
\hline Heart and great vessel function & 0.0 & 0.0 & 0.2 & 0.0 & 0.0 & 0.0 & 0.2 & 0.0 & 0.2 \\
\hline Lungs & 0.0 & 0.2 & 0.5 & 0.0 & 0.0 & 0.0 & 0.7 & 0.0 & 0.7 \\
\hline Abdomen & 0.0 & 2.5 & 2.9 & 0.5 & 0.2 & 0.5 & 4.4 & 0.7 & 5.9 \\
\hline Solid organs & 0.0 & 1.2 & 1.5 & 0.0 & 0.0 & 0.0 & 2.2 & 0.5 & 2.7 \\
\hline Hollow organs & 0.0 & 0.5 & 0.2 & 0.0 & 0.0 & 0.0 & 0.5 & 0.2 & 0.7 \\
\hline Solid and hollow organs* & 0.0 & 0.7 & 1.2 & 0.0 & 0.2 & 0.0 & 1.7 & 0.0 & 2.0 \\
\hline Natal tissue* & 0.0 & 0.0 & 0.0 & 0.5 & 0.0 & 0.5 & 0.0 & 0.0 & 0.5 \\
\hline Pelvis* & 0.0 & 6.9 & 0.2 & 0.0 & 1.7 & 0.5 & 4.2 & 0.7 & 7.1 \\
\hline Upper extremities* & 0.5 & 18.4 & 0.2 & 0.0 & 1.2 & 2.9 & 11.5 & 3.4 & 19.1 \\
\hline Clavicle* & 0.0 & 0.5 & 0.0 & 0.0 & 0.0 & 0.0 & 0.5 & 0.0 & 0.5 \\
\hline Humerus* & 0.0 & 1.5 & 0.2 & 0.0 & 0.0 & 0.7 & 1.0 & 0.0 & 1.7 \\
\hline Other long bones* & 0.2 & 7.1 & 0.0 & 0.0 & 0.5 & 1.0 & 4.9 & 1.0 & 7.4 \\
\hline Hand and fingers* & 0.0 & 1.2 & 0.0 & 0.0 & 0.2 & 0.0 & 0.5 & 0.5 & 1.2 \\
\hline Joints* & 0.2 & 8.1 & 0.0 & 0.0 & 0.5 & 1.2 & 4.7 & 2.0 & 8.3 \\
\hline Lower extremities* & 0.0 & 13.5 & 0.0 & 0.0 & 1.5 & 0.2 & 9.3 & 2.5 & 13.5 \\
\hline Femur & 0.0 & 3.7 & 0.0 & 0.0 & 0.7 & 0.2 & 2.0 & 0.7 & 3.7 \\
\hline Other long bones & 0.0 & 1.7 & 0.0 & 0.0 & 0.2 & 0.0 & 1.2 & 0.2 & 1.7 \\
\hline Joints & 0.0 & 8.1 & 0.0 & 0.0 & 0.5 & 0.0 & 6.1 & 1.5 & 8.1 \\
\hline Sensory organs & 0.2 & 2.0 & 0.7 & 0.0 & 0.5 & 0.0 & 0.7 & 1.7 & 2.9 \\
\hline Skin & 0.2 & 0.5 & 0.2 & 0.0 & 0.5 & 0.0 & 0.2 & 0.2 & 1.0 \\
\hline Eye* & 0.0 & 1.5 & 0.5 & 0.0 & 0.0 & 0.0 & 0.5 & 1.5 & 2.0 \\
\hline Neck (non-spinal)* & 0.0 & 0.2 & 0.0 & 0.0 & 0.0 & 0.0 & 0.2 & 0.0 & 0.2 \\
\hline Total & 6.9 & 74.5 & 17.6 & 1.0 & 12.7 & 5.6 & 65.7 & 15.9 & 100.0 \\
\hline
\end{tabular}

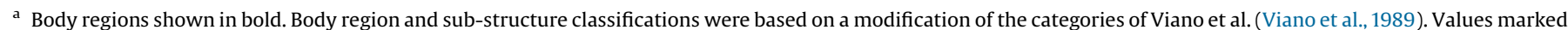
with asterisk are new additions to that taxonomy.

b Non-injurious volunteer studies or human observational studies.

c Human cell cultures or post-delivery, extra-fetal, natal tissue.

${ }^{\mathrm{d}}$ Includes living human and cadaver studies that used both male and female subjects, and animal studies.

biomechanics review of Viano et al. (1989). The overall body regions were the head, spine, thorax, abdomen, pelvis, upper extremity, lower extremity, sensory organs (skin and eyes), and neck (other than spine). Those body regions were then subdivided into anatomical structures, groups of anatomical structures, or functions (e.g., brain function; Table 3). Each paper was assigned to a single category. For the limited papers that spanned multiple categories, each was assigned based on the body structure for which the greatest amount of injury tolerance information was provided (for example, the body structure with the greatest sample size).

\subsection{Analysis}

Despite efforts to capture every reference meeting the selection criteria included in the Index, it is likely that some publications were missed either due to an absence of the search terms or due to reviewer error. It is also likely that appropriate papers exist outside of the Index, both within and outside of the automotive injury research field. Thus, the final review database was assumed to be a sample, rather than a census, of the papers available in the field. The results of the descriptive analysis are presented as percentages of the papers in the injury tolerance review database. Confidence intervals (95\%, assuming a simple random sample) were also calculated for the description of the general database characteristics (Table 2).

\subsection{Epidemiologic data}

Field injury distributions were drawn from the 1st global burden of disease (GBD) study of the World Health Organization (Murray and Lopez, 1996). The GBD data included a relative distribution of road traffic accident deaths, hospitalizations, and emergency ward visits across 33 injury categories, and across 5 different age groups. Those data covered three countries which met minimum data reporting requirements: Mauritius, Sweden, 
Table 4

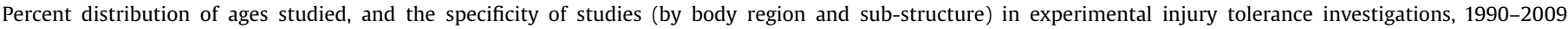
( $n=408$ studies).

\begin{tabular}{|c|c|c|c|c|c|c|c|c|c|}
\hline \multirow[t]{2}{*}{ Body regions and sub-structures ${ }^{a}$} & \multicolumn{5}{|l|}{ Age $^{\mathrm{b}}$} & \multicolumn{3}{|c|}{ Specificity } & \multirow[t]{2}{*}{ Total } \\
\hline & $0-22$ & $23-65$ & $>65$ & General $^{c}$ & Missing & General & Car driver or occupant & Pedestrian & \\
\hline Head & 3.2 & 4.4 & 3.4 & 7.1 & 1.7 & 15.0 & 0.7 & $\mathbf{0 . 0}$ & 15.7 \\
\hline Skull & 1.5 & 2.5 & 2.7 & 0.2 & 1.7 & 5.9 & 0.0 & 0.0 & 5.9 \\
\hline Face & 0.0 & 1.0 & 0.7 & 0.0 & 0.0 & 0.2 & 0.7 & 0.0 & 1.0 \\
\hline Brain structure & 0.0 & 0.2 & 0.0 & 0.5 & 0.0 & 0.7 & 0.0 & 0.0 & 0.7 \\
\hline Brain function & 1.7 & 0.7 & 0.0 & 6.4 & 0.0 & 8.1 & 0.0 & 0.0 & 8.1 \\
\hline Spine & 3.2 & 13.7 & 9.8 & 3.2 & 2.0 & 11.5 & 8.6 & $\mathbf{0 . 0}$ & 20.1 \\
\hline Vertebrae & 0.0 & 1.7 & 1.5 & 0.7 & 0.2 & 3.2 & 0.0 & 0.0 & 3.2 \\
\hline Ligaments & 0.2 & 1.2 & 0.7 & 0.5 & 0.0 & 1.5 & 0.2 & 0.0 & 1.7 \\
\hline Vertebrae and ligament structure & 2.9 & 10.8 & 7.6 & 1.0 & 1.2 & 5.6 & 8.1 & 0.0 & 13.7 \\
\hline Spinal cord structure & 0.0 & 0.0 & 0.0 & 1.0 & 0.0 & 0.7 & 0.2 & 0.0 & 1.0 \\
\hline Spinal cord function & 0.0 & 0.0 & 0.0 & 0.0 & 0.5 & 0.5 & 0.0 & 0.0 & 0.5 \\
\hline Thorax & 1.2 & 11.0 & 11.0 & 1.5 & 0.0 & 8.8 & 6.4 & 0.2 & 15.4 \\
\hline Ribcage & 0.7 & 8.8 & 9.1 & 1.0 & 0.0 & 5.9 & 6.1 & 0.2 & 12.3 \\
\hline Heart and great vessel structure & 0.2 & 2.2 & 2.0 & 0.0 & 0.0 & 2.0 & 0.2 & 0.0 & 2.2 \\
\hline Heart and great vessel function & 0.0 & 0.0 & 0.0 & 0.2 & 0.0 & 0.2 & 0.0 & 0.0 & 0.2 \\
\hline Lungs & 0.2 & 0.0 & 0.0 & 0.2 & 0.0 & 0.7 & 0.0 & 0.0 & 0.7 \\
\hline Abdomen & 0.2 & 1.7 & 2.0 & 2.7 & 1.0 & 3.7 & 2.2 & $\mathbf{0 . 0}$ & 5.9 \\
\hline Solid organs & 0.0 & 0.7 & 0.7 & 1.5 & 0.5 & 2.5 & 0.2 & 0.0 & 2.7 \\
\hline Hollow organs & 0.0 & 0.5 & 0.5 & 0.2 & 0.0 & 0.5 & 0.2 & 0.0 & 0.7 \\
\hline Solid and hollow organs & 0.2 & 0.5 & 0.7 & 1.0 & 0.0 & 0.2 & 1.7 & 0.0 & 2.0 \\
\hline Natal tissue & 0.0 & 0.0 & 0.0 & 0.0 & 0.5 & 0.5 & 0.0 & 0.0 & 0.5 \\
\hline Pelvis & 0.5 & 5.4 & 6.4 & 0.2 & 0.2 & 4.7 & 2.0 & 0.5 & 7.1 \\
\hline Upper extremities & 1.0 & 12.3 & 15.4 & 0.2 & 2.2 & 13.0 & 6.1 & 0.0 & 19.1 \\
\hline Clavicle & 0.0 & 0.5 & 0.5 & 0.0 & 0.0 & 0.5 & 0.0 & 0.0 & 0.5 \\
\hline Humerus & 0.0 & 1.0 & 1.2 & 0.2 & 0.2 & 1.5 & 0.2 & 0.0 & 1.7 \\
\hline Other long bones & 0.5 & 3.9 & 5.6 & 0.0 & 1.0 & 3.2 & 4.2 & 0.0 & 7.4 \\
\hline Hand and fingers & 0.0 & 0.5 & 0.5 & 0.0 & 0.7 & 1.0 & 0.2 & 0.0 & 1.2 \\
\hline Joints & 0.5 & 6.4 & 7.6 & 0.0 & 0.2 & 6.9 & 1.5 & 0.0 & 8.3 \\
\hline Lower extremities & 0.2 & 10.5 & 10.8 & 0.0 & 1.7 & 6.1 & 4.7 & 2.7 & 13.5 \\
\hline Femur & 0.0 & 2.7 & 3.2 & 0.0 & 0.5 & 2.7 & 0.5 & 0.5 & 3.7 \\
\hline Other long bones & 0.0 & 1.2 & 1.2 & 0.0 & 0.2 & 0.7 & 0.7 & 0.2 & 1.7 \\
\hline Joints & 0.2 & 6.6 & 6.4 & 0.0 & 1.0 & 2.7 & 3.4 & 2.0 & 8.1 \\
\hline Sensory organs & 0.2 & 0.5 & 0.5 & 0.7 & 1.5 & 2.2 & 0.7 & 0.0 & 2.9 \\
\hline Skin & 0.2 & 0.2 & 0.2 & 0.2 & 0.2 & 0.7 & 0.2 & 0.0 & 1.0 \\
\hline Eye & 0.0 & 0.2 & 0.2 & 0.5 & 1.2 & 1.5 & 0.5 & 0.0 & 2.0 \\
\hline Neck (non-spinal) & 0.0 & 0.2 & 0.2 & 0.0 & 0.0 & 0.0 & 0.2 & 0.0 & 0.2 \\
\hline Total & 9.8 & 59.8 & 59.6 & 15.7 & 10.3 & 65.0 & 31.6 & 3.4 & 100.0 \\
\hline
\end{tabular}

and Chile. For comparison purposes, the injury and age categories of the GBD data were aggregated to correspond (as closely as possible) with the body regions and age groups of the injury tolerance review (Table 5). In addition to the GBD injury distribution description, specific field data studies are included in the discussion section where appropriate.

\section{Results}

Including repeats and search overlaps, the search terms listed in Table 2 resulted in a total of approximately 88,000 returned Index entries. Of those, 77,000 entries were within the time period of interest. From the initial review, approximately 1000 of those entries were selected for possible inclusion in the database. After the elimination of repeat entries and final review, 408 papers were identified that met the selection criteria (this bibliography is available from the authors by request). Of those, $94 \%$ were peerreviewed articles from journals or conference proceedings, or were books, book chapters, or theses. The remainder were non-peerreviewed conference papers or reports.

Table 2 summarizes select information from the papers in the final review database; Tables 3 and 4 present their distribution regarding body region, model used, gender, age, and road-user specificity. For the sake of simplicity, Tables 3 and 4 do not present the $95 \%$ confidence intervals (these are available from the authors by request). The body region with the most papers was the spine (20\% of the papers, 95\% C.I.: 16.2, 24.0), followed by the upper extremities (19\%: $15.3,22.9)$, the thorax $(15 \%: 11.9,18.9)$, the head (16\%: 12.2, 19.2), and the lower extremities (14\%: 10.2, 16.8). Of all the papers, $75 \%$ used cadavers or cadaver tissue as experimental models. Eighteen percent animal models; 7\% living human models; and $1 \%$ other human tissue were used. Cadaver models were used to study skeletal injury (osseous and ligamentous) in almost every body region, in addition to injury to the abdominal viscera (both solid and hollow organs), and structural injury to the sensory organs of the skin and the eye. Animal models were predominantly 
used to study soft tissue or physiological injuries such as brain injury ( $42 \%$ of the animal studies), abdominal injury (17\%), spinal cord injury (8\%), and heart or lung injury (1.5\%). Living human models were used predominantly in non-injurious studies of spinal mechanics (68\% of living human studies), or observational studies of concussion in athletes (10\%). Most of the studies (66\% of all papers) were applicable to either gender, either through the use of both male and female human subjects or through the use of animal models. Thirteen percent used male subjects only, and $6 \%$ used female subjects only (gender information was missing from the remainder). Sixty percent of the studies used subjects in the age range $23-65$, and $60 \%$ used subjects over the age of 65 . Ten percent used subjects representing the $0-22$ year age range.

Most of the studies (65\%) were generally applicable, providing tolerance information that was not specific to a particular injury causation scenario. Thirty-two percent specifically targeted loading scenarios experienced by automobile occupants in collisions. Only $3 \%$ specifically targeted the loading scenario of a pedestrian being struck by a vehicle.

Table 5 presents the distribution of injured body regions among road traffic accident deaths, hospitalizations, and emergency ward visits identified by the 1 st global burden of disease study (Murray and Lopez, 1996). Those results have been aggregated to allow comparison with the body region categories and age groups used in the current study. The head was the most injured region in the youngest (age $0-14$ ) and middle (15-59) age groups. The head accounted for $48 \%$ of injuries among the youngest age group, with this proportion decreasing with increasing age. Compared to the youngest and middle age groups, the oldest age group exhibited a greater relative frequency of spinal, thoracic, pelvic, and lower extremity injuries, with the lower extremity (30\%) surpassing the head $(28 \%)$ as the most frequently injured body region.

\section{Discussion}

In this study, we have summarized 20 years of injury tolerance research as applied to the prediction and prevention of automotive injuries. The volume of original research represents a substantial increase from the (approximately) 145 injury biomechanics publications reviewed by Viano et al. (1989). While some of the knowledge deficiencies noted by Viano et al. (1989) have been addressed in the intervening years, several areas of injury tolerance remain understudied. Demographic and road use changes in the last 20 years have also prompted new priorities and challenges not envisaged by the previous review. In the sections below we discuss some of the areas in which success has

Table 5

Percent distributions of road traffic accident deaths, hospitalizations, and emergency ward visits by age. (adapted from Table 4.3 of the 1st global burden of disease study of the World Health Organization (Murray and Lopez, 1996) . $^{\text {. }}$

\begin{tabular}{lrrr}
\hline & Age & & \\
\cline { 2 - 4 } & $0-14$ & $15-59$ & $60+$ \\
\hline Head & 48.0 & 36.1 & 27.6 \\
Spine & 0.9 & 6.6 & 6.1 \\
Thorax & 0.2 & 2.7 & 8.1 \\
Pelvis & 0.7 & 2.3 & 3.9 \\
Upper extremities & 15.0 & 11.1 & 10.6 \\
Lower extremities & 15.3 & 18.8 & 29.9 \\
Sensory organs & 0.1 & 0.2 & 0.1 \\
Other & 20.0 & 22.3 & 14.0 \\
Total & & & 100.0
\end{tabular}

\footnotetext{
a The age and injury category groups were aggregated into categories most closely analogous to those used in the current study (Tables 2 and 4).

b Injury type definitions that could not be aggregated into one of the body region definitions used in the current study. This includes, but is not limited to, non-spinal neck injuries, pelvis injuries, and abdominal injuries.
}

been gained in injury tolerance research in addition to areas where more work is needed, and how this research relates to the injury prevention priorities observed in the epidemiologic data.

\subsection{Head injury}

The head remains the most frequently injured body region resulting in death or hospitalization in motor-vehicle collisions in the field (Table 5). Unfortunately, significant challenges remain in understanding the sources and tolerances of physiological head injury. As noted by Viano et al. (1989), the tolerance of gross skull fracture has been relatively well established. Conversely, Viano et al. (1989) highlighted physiological head injury as an area for which little or no knowledge was available. As a result, substantial research in the last 20 years has focused on brain injury, including $52 \%$ of the head injury papers reviewed here. Most of those (33\% of the head studies) investigated brain injury following controlled impact in animal models, identified either through functional or histological changes. Fourteen percent of the head papers studied the response of neural cell cultures (mostly from animal sources) to mechanical loading such as stretching (Bottlang et al., 2007; Cater et al., 2006; Elkin and Morrison, 2007; Geddes and Cargill, 2001; Geddes-Klein et al., 2006; LaPlaca et al., 2005; Morrison et al., 2000, 2003; Smith et al., 1999). A further $3 \%$ of the head papers studied concussive injury in American football players resulting from impacts in real game play (Duma et al., 2005; Zhang et al., 2004). These research trends are encouraging given the previous lack of information on functional brain injury (Viano et al., 1989), and given the enduring presence of functional brain injury in the field (Tagliaferri et al., 2009). The next challenge lies in interpreting the knowledge gained from animal, cell-culture, and observational sports studies to develop injury prediction and prevention techniques in humans in injury causation environments in the field. Computational modeling techniques may provide the bridge in this effort (Zhang et al., 2004; Ueno et al., 1995; Huang et al., 2000; Cavanaugh et al., 1993; Bain et al., 2003; Zhang et al., 2001a,b, 2002; Takhounts et al., 2003, 2008), with the available studies providing input and validation information for tissue-level injury prediction with those models.

\subsection{Spinal injury}

The spine was the most commonly studied body region in the papers reviewed. The majority of those (59\% of the spine studies) investigated the mechanics of the cervical spine. This focus is likely due to a combination of the grave sequelae often associated with severe cervical spine injury, and the high frequency of lowerseverity cervical spine injury (e.g., "Whiplash Associated Disorder", WAD (Siegmund et al., 2009)) reported in some countries. In contrast to the GBD data (Table 5) (Murray and Lopez, 1996), WADtype neck sprains are the most common automobile-related injuries treated in hospital emergency rooms in the U.S. (Quinlan et al., 2004). Because WAD symptoms are often not associated with acute fracture or dislocation, it has been uniquely difficult to determine both the anatomical source of the pain, and the physical mechanisms of the injury in collisions. The WAD-type studies reviewed here focused on either characterizing the kinematics (the motion of the anatomical structures) of the spine during a lowspeed rear impact, or focused on determining potential anatomical sources of pain associated with WAD. As with brain injury, the next challenge lies with combining available knowledge on spine kinematics with the knowledge on the anatomical and physiological sources of injury to facilitate the prediction and prevention of injury in the field. 
Spinal cord injuries, although relatively rare, are of concern due to the severe long-term neurological deficits that can occur. Unfortunately, both Viano et al. (1989) and the current review found information on spinal cord injury tolerance lacking. As with other physiological central nervous system injuries, this lack of information is likely due to a lack of a convenient model for study. Future efforts should include expanding this knowledge base, as well as developing methods to translate the knowledge gained with animal models to the prediction of injury in humans.

Only $8 \%$ of the spine papers studied the structure of the lumbar or thoracic spines. While this may be consistent with the relative frequency of injuries among automobile occupants (Hassan et al., 1996; Yoganandan et al., 1989), thoracic and lumbar spine injuries represent relatively common injuries among other road users (e.g., motorcyclists (Robertson et al., 2002)). As efforts to protect different types of road users progress, it will become necessary to refine our knowledge of the tolerance and consequences of injury to these regions.

\subsection{Upper extremities}

Consistent with their high frequency in the field, the upper extremities were one of the most studied body regions in the papers reviewed. This represents an addition to the previous state of knowledge, as the upper extremities were not even included as a distinct body region of interest in the previous review (Viano et al., 1989). This research focus appears to have resulted from two concerns: study of upper extremity interactions with deploying airbags; and increased study of injury tolerances in side-impact collisions. Twenty-nine percent of the upper-extremity studies investigated the bending tolerance of the forearm, largely drawing from efforts to avoid injury to the forearm from close-proximity deployment of airbags. Thirty percent of the upper extremity papers focused on the shoulder joint, intending to simulate shoulder loading during a side-impact automobile collision. While injury to the shoulder may not necessarily be life-threatening, recent studies have indicated that structural failure of the shoulder in side impacts can lead to increased injury risk to the thorax (Subit et al., 2010). The shoulder has traditionally served as a target for occupant loading in a side collision (considered stronger than the other body structures), but efforts to increase the loading borne by the shoulder must be balanced with its failure tolerance. Future efforts should include refining our understanding of shoulder and clavicle tolerances under various loading scenarios, especially as a function of increased fragility with age.

\subsection{Lower extremities}

Also consistent with their frequency in the field, a substantial number (14\%) of the reviewed papers studied the injury tolerance of the lower extremities. As information on knee, ankle, and hip injuries were previously lacking (Viano et al., 1989), most of the lower extremity papers (61\%) concerned joint injuries. Around half of those ( $28 \%$ of the lower extremity papers) studied injury to the ankle or the foot/ankle complex (Funk et al., 2002; Yoganandan et al., 1997; Parenteau et al., 1998; Rudd et al., 2004; McKay and Bir, 2009), and around half (30\%) studied injury to the knee (Bose et al., 2008; Jones et al., 1995; Atkinson and Haut, 1995, 2001; Balasubramanian et al., 2004). Most of the foot/ankle papers studied loading conditions similar to what would occur in the hind foot and ankle of a person in a frontal car crash, either under axial compression of the ankle or under forced dorsiflexion. While such injuries are not necessarily life-threatening, they are considered an important source of long-term disability following collisions (MacKenzie et al., 2002).
Due to the use of cadaver models, few studies considered the effects of active musculature on lower extremity injury tolerances (Soni et al., 2010). While it may be difficult to investigate experimentally, it may be possible to explore the effects of active musculature using computer models (Chang et al., 2008).

\subsection{Abdominal injury}

The study of abdominal injury tolerance has been hampered by limitations of the cadaver model for studying soft tissue injury. While cadaveric tissue can be readily used to study fracture tolerance in bone, post-mortem autolysis quickly degrades the abdominal viscera. Lack of circulation also limits the study of visceral contusions and small lacerations that are identified by bleeding. Most of the abdomen studies (63\%) used animal models to bypass these limitations. While providing valuable information, those studies were also limited by anatomical differences between humans and the animal models used. Future efforts should include translational research to apply the results of animal studies and in vitro experiments to the prediction of injury in the field.

A limited number of studies investigated the failure tolerance of post-delivery, extra-fetal natal tissue (the chorioamnion (Pressman et al., 2002) and the fetal membrane (Oyen et al., 2004)). Motor vehicle collisions are the leading cause of traumatic fetal death (Weiss et al., 2001; Weiss, 2001). Despite this, there remains little information on the mechanisms or tolerances to injury among pregnant women. Again, this is largely due to a lack of an appropriate model for the study of maternal and natal injury. The existing studies are a first-step in this effort, but more knowledge is needed regarding the specific biomechanics related to fetal injury or death (for example, from placental abruption). The largest challenge will come in combining observations from individual tissues to gain insight into the protection of the pregnant abdomen as a whole. As with functional brain injury, computer modeling may ultimately serve as the bridge in this effort.

\subsection{Pelvis injury}

Seven percent of the papers reviewed studied the injury tolerance of the pelvis. Most of those studied lateral impacts of the pelvis (Beason et al., 2003; Dakin et al., 2001; Etheridge et al., 2005; Leport et al., 2007), applicable either to vehicle occupants in sideimpact automobile collisions, or pedestrians struck by a vehicle from the side. A limited number of papers studied frontal impacts to the pelvis (Salzar et al., 2006; Uriot et al., 2006), or the fracture tolerance of the acetabulum resulting from an impact to the knee (Rupp et al., 2002, 2003; Yoganandan et al., 2001). Absent from those papers is a study of the tolerance of the pelvis to loading by a lap belt (in a normal geometry) in a frontal impact automobile collision. The pelvis has long been regarded by automobile restraint designers as a relatively strong structure that can serve as a target for applying restraining force to the body. The use of lapbelt pretensioners (devices that increase the tension in the lap-belt at the initiation of a collision) (Walz, 2004) may increase the amount of restraining force applied to the pelvis. Without understanding the tolerance of the pelvis to lap-belt loading, new injury patterns could emerge as new restraint designs are implemented in the field.

\subsection{Gender}

The majority of the studies were applicable to either gender. Of the studies that were gender-specific, $69 \%$ were specific to males and $31 \%$ (including the studies of natal tissue) were specific to females. This is consistent with the gender-distribution in the field 
- approximately $68 \%$ of fatally injured and $61 \%$ of hospitalized traffic-injury victims in the U.S. are male (Finkelstein et al., 2006). For most of the male-specific studies, it is unknown if that gender was targeted specifically or if the disparity resulted from limitations in the subject pool. In contrast, most (65\%) of the female-specific studies either targeted females as the study group, or performed tests on female-specific tissue (i.e., natal tissue). This represents a significant addition to the previous literature - Viano et al. (1989) noted that knowledge on injury tolerances specific to females were practically non-existent prior to 1989. While it may not be necessary to discern male and female-specific injury tolerances for all body regions, it would be wise to continue considering gender as a potential confounding factor when studying age-related skeletal injury tolerances given differences in the onset and progression of osteoporosis (Melton et al., 1997), and potential effects of differences in musculature and anthropometry.

\subsection{Age}

As our knowledge of injury biomechanics becomes more refined, increasing attention is paid to injury tolerance as a function of age. Aging has profound effects on the morphology, geometry, and mechanics of tissues, from tissue growth and skeletal ossification in childhood and adolescence through osteoporosis and bone loss with advanced age. As a result, many recent efforts have focused on differentiating injury tolerances based on age to identify specialized injury prevention strategies. These differentiation efforts have focused on two distinct age groups - the elderly and children.

Several recent studies have investigated the effects of advanced age on injury tolerances. Those have largely focused on the chest and ribcage (Kent et al., 2005a; Kent and Patrie, 2005; Laituri et al., 2005), driven by the age-related shift toward serious chest injury in automobile collisions in the field (Table 5) (Kent et al., 2005b). As shown in Table 4, there appears to be a wealth of information on the injury tolerance of elderly tissue for almost every body region. Due to the reliance on cadaver models, $80 \%$ of the studies with age information available (excluding those with generally-applicable animal models) included experiments with subjects or tissue over the age of 65 . As a result, our general knowledge of skeletal injury tolerances includes - and may be skewed toward - the elderly.

Despite this tendency toward the study of elderly subjects, many injury biomechanics studies specifically screen their subjects for age-related pathologies such as osteoporosis in an attempt to produce results more representative of the general population. As a result, many such studies may exclude the effects of common pathologies that may affect injury risk in advanced age.

In addition, there remains a dearth of information on injury tolerances of younger populations, especially pediatric subjects. Of the studies with age information available, only $13 \%$ performed experiments with subjects under the age of twenty three. Only 5\% specifically targeted the pediatric population. As with other limitations observed in this review, this is largely due to a lack of appropriate models to study pediatric injury. Pediatric cadaver studies are limited due to a rarity of donated tissue, and may be confounded by a general reluctance to pursue this line of research. Such studies do exist (Ouyang et al., 2006; Benz et al., 1992; Kent et al., 2009; Coats and Margulies, 2006; Holck, 2005), but are often limited to a very small sample size - sometimes including only a single subject (Kent et al., 2009). As a result, novel solutions are needed for the study of pediatric injury tolerances for all of the body regions, even the structures that can be readily studied with cadaver models in adults. Some success has been achieved with age-specific animal models to study abdominal tolerance to lap belt loading (Kent et al., 2008), skull fracture (Margulies and
Thibault, 2000), and traumatic brain injury (Raghupathi and Margulies, 2002). Some success has also been achieved through observational studies with pediatric subjects - for example, an observational study of chest loading during clinical cardio-pulmonary resuscitation of pediatric patients (Arbogast et al., 2006). Improving our knowledge will require continuing experimental studies with all available models. This should be augmented by an improved understanding of the basic principles of injury biomechanics (i.e., material-level failure mechanics), how those characteristics change with age, and the development of improved translational models to predict injury based on those fundamental principles.

\subsection{Road-user specificity}

A majority of the studies (64\%) resulted in injury tolerance information that was generally applicable, not specific to a particular injury-causation scenario. Thirty-two percent studied injury tolerances in scenarios specific to automobile occupants involved in collisions. Of those, most (approximately 66\% of the occupant-specific studies) studied injury tolerances in simulated frontal-impact collisions. Eighteen percent studied side-impact collisions, and $16 \%$ studied rear-impact collisions. None of the studies investigated injury tolerances specifically in rollover scenarios, likely due to the difficulty of reproducing rollover collisions in a controlled laboratory environment. As rollovers account for up to $25 \%$ of passenger vehicle deaths in the U.S. (Brumbelow et al., 2009), injury tolerances and mechanisms in the rollover environment represent a topic for future study.

Only $3 \%$ of the studies specifically targeted the scenario of a pedestrian struck by a vehicle. No studies (to the authors' knowledge) specifically investigated injury tolerances characteristic to either powered-two-wheeler users (PTW; motorcycles, mopeds, and scooters), or bicycle riders struck by vehicles. Together, pedestrians, PTW users, and bicyclists represent a category known as "vulnerable road users". Vulnerable road users represent a significant road safety problem, accounting for approximately half of road traffic fatalities worldwide (WHO, 2009). This is likely to worsen, given trends toward increasing PTW sales in both high-income and low-income countries (Segui-Gomez and Lopez-Valdes, 2007; Iyer and Badami, 2007). Despite technological challenges, substantial engineering research has concerned developing countermeasures to protect vulnerable road users. This has included altering the external of vehicles to protect pedestrians (Crandall et al., 2002), and improved roadside barriers to protect PTW users (Peldschus et al., 2007). Such efforts have been limited, however, by a lack of knowledge on injury tolerances characteristic to vulnerable road user collision scenarios (Peldschus et al., 2007; Kerrigan et al., 2008). Continuing improvement of vulnerable road user safety will require epidemiologic study to identify where additional technological interventions are possible, combined with injury tolerance research to identify design targets for those scenarios.

\subsection{Limitations and future work}

This study sought to provide a systematic review of experimental studies relating to injury tolerance research published from 1990-2009 compared to the previous state of knowledge and to injury characteristics observed in the epidemiologic data. Some limitations arise from the inclusion/exclusion criteria of this study. First, for practical purposes this study was limited to publications written in English. Some papers likely exist in other languages native to countries with an automobile safety research presence (e.g., Japan, Germany, France). Second, this study was limited to publications included in a specific in-house reference index, 
treated as a random sample of papers available in the field. There is no evidence to suggest that there is any selection bias in this index that would have affected the outcome of this study, although it is conceivable that some unknown selection bias may exist.

This study was also limited to papers that included new experimental data on injury tolerances using biological models. This represents a small portion of the injury biomechanics and automotive safety research field, which also includes efforts to predict injury with artificial models (either physical models or computer simulations), and studies into the sub-failure mechanical properties of biological tissues. As a result, this study is not necessarily representative of the total research effort in injury biomechanics and automobile safety, but instead represents the field's achievements and limitations in experimental injury tolerance research. Future work should include an examination of the injury biomechanics field as a whole to identify research priorities and opportunities for improvement.

While information is available for some individual countries or collision scenarios, it is difficult to ascertain global injury distributions and research priorities in motor vehicle safety. The GBD data referenced here represent the broadest and most international work to date, but are limited in the time-period covered, the countries covered, and the specificity of the information available (in both gender and road-user type). It is anticipated that current, ongoing efforts will provide a more precise description of the world-wide injury distributions in the near future (Bhalla, 2011). Even with improved injury frequency information, however, determining research priorities is not necessarily clear. Metrics such as injury severity, risk of death, long-term disability, DALYs lost, and economic burden may be also considered.

In addition, injury biomechanics research priorities are usually focused on areas for which an engineering solution is indicated. A subset of the injuries occurring in the field (either by population, road user type, or collision type) may be best addressed through alternative means such as education, infrastructure, or policy. Even in such cases, however, limitations in the implementation feasibility of primary prevention strategies may still lead to opportunities for reduction in injury risk or severity through passive protection and crashworthiness engineering. Research priorities should also be formed in the context of new developments in safety technology. For example, technological developments such as brake assist and electronic stability control will likely change the distribution of collisions and injuries in the field reshaping the passive protection goals of future researchers. Further advances in vehicle autonomy will likely bring on new challenges (such as automated decision making for injury risk minimization, or automatic injury risk assessment for the direction of emergency response) which may require an increasingly precise understanding of injury tolerances for the prediction of risk for specific occupant demographics in diverse collision scenarios.

Research trends may also be influenced (intentionally or otherwise) by geographic factors. Although not specifically tabulated in this study, the selection criteria of English as the publication language likely limited the observance of research performed in countries where English is not the primary language of technical publication. Future work should include an examination research performed in developing countries and countries where English is not the primary language of technical publication, as well as an examination of the origin and evolution of research priorities in those countries to identify new high-impact opportunities for study and intervention.

Finally, the analysis presented here is a general overview of the efforts of the field in the last twenty years, as reflected in the publication record. It is beyond the scope of this manuscript to comment on the quality of the research performed, or on the accuracy or precision of the knowledge gained in those studies.
Examining the specific knowledge gained - including the specific injury tolerances and the fields needing improvement - requires careful, in-depth study of the papers related to one's topic of interest. It is the authors' intention that this study serves as a spring board for future discussions, and for in-depth study of the topics (body regions, injury types, afflicted populations, and injury causation scenarios) for which limited knowledge is available.

\section{Conclusions}

Unintentional injuries resulting from automobile collisions are a significant threat to public health world-wide. Reducing these injuries requires complementary, coordinated efforts in injury epidemiology and injury tolerance research. Evidence on the burden of injuries should be contrasted with fundamental biomechanics knowledge to set priorities for intervention design, policy implementation, and for the prioritization of future research.

The past 20 years have seen considerable advances in injury tolerance research. Since 1989, substantial knowledge has been gained regarding the injury tolerances of the upper extremity, the joints and long-bones of the lower extremity, the face and eyes, the thorax, the abdomen, and the structure of the spine. Considerable research has also investigated differential injury tolerances with advanced age, and some effort has focused on identifying injury tolerances specific to females.

There remain, however, considerable gaps in knowledge - most resulting from experimental difficulties and limitations of the models available for study. Because of a reliance on cadaver models, most research has involved studying the injury tolerance of the skeletal system, including studies of almost every bone in the body. Unfortunately, cadavers are limited in their ability to model soft-tissue and physiological injury. Some recent investigations using animal models, cell cultures, and observational studies in humans have each provided valuable information on different aspects of central nervous system and soft-tissue injury. In addition to continuing to identify novel models for studying physiological injury, future efforts should include translational research to apply those findings to the prediction and prevention of physiological injury in humans - likely with the aid of computer models.

In contrast to middle-age and elderly adults, information on injury tolerance among the young adult and pediatric populations is severely lacking. Like with physiological injury, this is largely due to a lack of models for study. Future efforts should include identifying appropriate models (and translational methods) for the study of pediatric injury biomechanics, and maximizing the information gained from the limited pediatric tissue donation resources that are available.

Finally, while most of the reviewed studies resulted in fundamental injury tolerance information that was generally applicable, several studies did investigate scenarios specific to an automobile occupant involved in a collision. Those studies investigated injury tolerances in frontal, lateral, and rear-impact collisions. Given their prevalence in the field, future efforts should include exploring injury mechanisms and tolerances in vehicle rollover scenarios, and improving our understanding of tolerances and protection of vulnerable road users.

\section{Acknowledgements}

This work was supported in part by a Whitaker International Scholars Grant for post-doctoral biomedical engineering research. This work was also supported in part by internal funds from the European Center for Injury Prevention at the Universidad de Navarra, the University of Virginia Center for Applied Biomechanics, and the French Institute of Sciences and Technology for 
Transport, Development and Networks. The injury biomechanics literature index used in this study was developed, and is maintained, by the University of Virginia Center for Applied Biomechanics.

\section{References}

Arbogast, K.B., Maltese, M.R., Nadkarni, V.M., Steen, P.A., Nysaether, J.B., 2006 Anterior-posterior thoracic force-deflection characteristics measured during cardiopulmonary resuscitation: comparison to post-mortem human subject data. Stapp Car Crash J. 50, 131-145.

Atkinson, P.J., Haut, R.C., 1995. Subfracture insult to the human cadaver patellofemoral joint produces occult injury. J. Orthop. Res. 13, 936-944.

Atkinson, P.J., Haut, R.C., 2001. Impact responses of the flexed human knee using a deformable impact interface. J. Biomech. Eng. 123, 205-211.

Bain, A.C., Shreiber, D.I., Meaney, D.F., 2003. Modeling of microstructural kinematics during simple elongation of central nervous system tissue. J. Biomech. Eng. 125, 798-804.

Balasubramanian, S., Beillas, P., Belwadi, A., et al., 2004. Below knee impact responses using cadaveric specimens. Stapp Car Crash J. 48, 71-88.

Beason, D.P., Dakin, G.J., Lopez, R.R., Alonso, J.E., Bandak, F.A., Eberhardt, A.W., 2003. Bone mineral density correlates with fracture load in experimental side impacts of the pelvis. J. Biomech. 36, 219-227.

Benz, G., Kallieris, D., Daum, R., Zachariou, Z., 1992. Does lateral bending lead to bowing fracture in an infantile lower arm? Eur. J. Pediatr. Surg. 2, 177-179.

Bhalla, K., 2011. Global burden of disease injury expert group. http://sites.google. com/site/gbdinjuryexpertgroup (accessed 28.02.11).

Bose, D., Bhalla, K.S., Untaroiu, C.D., Ivarsson, B.J., Crandall, J.R., Hurwitz, S., 2008 Injury tolerance and moment response of the knee joint to combined valgus bending and shear loading. J. Biomech. Eng. 130, 031008.

Bottlang, M., Sommers, M.B., Lusardi, T.A., Miesch, J.J., Simon, R.P., Xiong, Z.G., 2007. Modeling neural injury in organotypic cultures by application of inertia-driven shear strain. J. Neurotrauma 24, 1068-1077.

Brumbelow, M.L., Teoh, E.R., Zuby, D.S., McCartt, A.T., 2009. Roof strength and injury risk in rollover crashes. Traffic Inj. Prev. 10, 252-265.

Cater, H.L., Sundstrom, L.E., Morrison III, B., 2006. Temporal development of hippocampal cell death is dependent on tissue strain but not strain rate. J. Biomech. 39, 2810-2818.

Cavanaugh, J., Zhu, Y., Huang, Y., King, A., 1993. Injury and response of the thorax in side impact cadaver tests, Stapp Car Crash Conference, pp. 37 Paper No. 933127.

Chandran, A., Hyder, A.A., Peek-Asa, C., 2010. The global burden of unintentional injuries and an agenda for progress. Epidemiol. Rev. 32, 110-120.

Chang, C.Y., Rupp, J.D., Kikuchi, N., Schneider, L.W., 2008. Development of a finite element model to study the effects of muscle forces on knee-thigh-hip injuries in frontal crashes. Stapp Car Crash J. 52, 475-504.

Coats, B., Margulies, S.S., 2006. Material properties of human infant skull and suture at high rates. J. Neurotrauma 23, 1222-1232.

Crandall, J.R., Bhalla, K.S., Madeley, N.J., 2002. Designing road vehicles for pedestrian protection. BMJ 324, 1145-1148.

Dakin, G.J., Arbelaez, R.A., Molz, F.J., Alonso, J.E., Mann, K.A., Eberhardt, A.W., 2001. Elastic and viscoelastic properties of the human pubic symphysis joint: effects of lateral impact joint loading. J. Biomech. Eng. 123, 218-226.

Duma, S.M., Manoogian, S.J., Bussone, W.R., et al., 2005. Analysis of real-time head accelerations in collegiate football players. Clin. J. Sport Med. 15, 3-8.

Elkin, B.S., Morrison III, B., 2007. Region-specific tolerance criteria for the living brain. Stapp Car Crash J. 51, 127-138.

Etheridge, B.S., Beason, D.P., Lopez, R.R., Alonso, J.E., McGwin, G., Eberhardt, A.W., 2005. Effects of trochanteric soft tissues and bone density on fracture of the female pelvis in experimental side impacts. Ann. Biomed. Eng. 33, 248-254.

Finkelstein, E.A., Corso, P.S., Miller, T.R., 2006. The Incidence and Economic Burden of Injuries in the United States. Oxford University Press.

Franklyn, M., Peiris, S., Huber, C., Yang, K.H., 2007. Pediatric material properties: a review of human child and animal surrogates. Crit. Rev. Biomed. Eng. 35, 197-342.

Funk, J.R., Srinivasan, S.C., Crandall, J.R., et al., 2002. The effects of axial preload and dorsiflexion on the tolerance of the ankle/subtalar joint to dynamic inversion and eversion. Stapp Car Crash J. 46, 245-265.

Geddes, D.M., Cargill, R.S., 2001. An in vitro model of neural trauma: device characterization and calcium response to mechanical stretch. J. Biomech. Eng. 123, 247-255.

Geddes-Klein, D.M., Schiffman, K.B., Meaney, D.F., 2006. Mechanisms and consequences of neuronal stretch injury in vitro differ with the model of trauma. J. Neurotrauma 23, 193-204.

Hassan, A.M., Parkin, S., Mackay, M., 1996. Spinal injuries in car collisions. 40th Proceedings Association for the Advancement of Automotive Medicine .

Holck, P., 2005. What can a baby's skull withstand? Testing the skull's resistance on an anatomical preparation. Forensic Sci. Int. 151, 187-191.

Huang, H.M., Lee, M.C., Lee, S.Y., Chiu, W.T., Pan, L.C., Chen, C.T., 2000. Finite element analysis of brain contusion: an indirect impact study. Med. Biol. Eng. Comput. 38, 253-259.

Iyer, N.V., Badami, M.G., 2007. Two-wheeled motor vehicle technology in India: evolution, prospects and issues. Energy Policy 35, 4319-4331.

Jones, R.S., Nawana, N.S., Pearcy, M.J., et al., 1995. Mechanical properties of the human anterior cruciate ligament. Clin. Biomech. (Bristol, Avon) 10, 339-344.
Kent, R., Patrie, J., 2005. Chest deflection tolerance to blunt anterior loading is sensitive to age but not load distribution. Forensic Sci. Int. 149, 121-128.

Kent, R., Lee, S.H., Darvish, K., et al., 2005a. Structural and material changes in the aging thorax and their role in crash protection for older occupants. Stapp Car Crash J. 49, 231-249.

Kent, R., Henary, B., Matsuoka, F., 2005b. On the fatal crash experience of older drivers. 49th Proceedings Association for the Advancement of Automotive Medicine .

Kent, R., Stacey, S., Kindig, M., et al., 2008. Biomechanical response of the pediatric abdomen, Part 2: injuries and their correlation with engineering parameters. Stapp Car Crash J. 52, 135-166.

Kent, R., Salzar, R., Kerrigan, J., et al., 2009. Pediatric thoracoabdominal biomechanics. Stapp Car Crash J. 53, 373-401.

Kerrigan, J.R., Crandall, J.R., Deng, B., 2008. A comparative analysis of the pedestrian injury risk predicted by mechanical impactors and post mortem human surrogates. Stapp Car Crash J. 52, 527-567.

Kopits, E., Cropper, M., 2003. Traffic fatalities and economic growth. Policy Research Working Paper Number 3035. The World Bank, Washington D.C.

Laituri, T.R., Prasad, P., Sullivan, K., Frankstein, M., Thomas, R.S., 2005. Derivation and Evaluation of a Provisional, Age-dependent, AIS3+ Thoracic Risk Curve for Belted Adults in Frontal Impacts. Society of Automotive Engineers.

LaPlaca, M.C., Cullen, D.K., McLoughlin, J.J., Cargill, R.S., 2005. High rate shear strain of three-dimensional neural cell cultures: a new in vitro traumatic brain injury model. J. Biomech. 38, 1093-1105.

Leport, T., Baudrit, P., Trosseille, X., Petit, P., Palisson, A., Vallancien, G., 2007. Assessment of the pubic force as a pelvic injury criterion in side impact. Stapp Car Crash J. 51, 467-488.

MacKenzie, E.J., Sacco, W.J., Luchter, S., et al., 2002. Validating the functional capacity index as a measure of outcome following blunt multiple trauma. Qual. Life Res. 11, 797-808.

Margulies, S.S., Thibault, K.L., 2000. Infant skull and suture properties: measurements and implications for mechanisms of pediatric brain injury. J. Biomech. Eng. 122, 364-371.

McKay, B.J., Bir, C.A., 2009. Lower extremity injury criteria for evaluating military vehicle occupant injury in underbelly blast events. Stapp Car Crash J. 53, 229-249.

Melton, L.J., Thamer, M., Ray, N.F., et al., 1997. Fractures attributable to osteoporosis: report from the National Osteoporosis Foundation. J. Bone Miner. Res. 12, 16-23.

Morrison III, B., Meaney, D.F., Margulies, S.S., McIntosh, T.K., 2000. Dynamic mechanical stretch of organotypic brain slice cultures induces differential genomic expression: relationship to mechanical parameters. J. Biomech. Eng. $122,224-230$

Morrison III, B., Cater, H.L., Wang, C.C., et al., 2003. A tissue level tolerance criterion for living brain developed with an in vitro model of traumatic mechanical loading. Stapp Car Crash J. 47, 93-105.

Murray, C.J.L., Lopez, A.D., 1996. The Global Burden of Disease: A Comprehensive Assessment of Mortality and Disability From Disease, Injuries, and Risk Factor in 1990 and Projected to 2020. In: Murray, C.J.L., Lopez, A.D. (Eds.), Harvard University Press, Cambridge, MA.

Murray, C.J., Lopez, A.D., 1997. Alternative projections of mortality and disability by cause 1990-2020: global burden of disease study. Lancet 349, 1498-1504.

Ouyang, J., Zhao, W., Xu, Y., Chen, W., Zhong, S., 2006. Thoracic impact testing of pediatric cadaveric subjects. J. Trauma 61, 1492-1500.

Oyen, M.L., Cook, R.F., Calvin, S.E., 2004. Mechanical failure of human fetal membrane tissues. J. Mater. Sci. Mater. Med. 15, 651-658.

Parenteau, C.S., Viano, D.C., Petit, P.Y., 1998. Biomechanical properties of human cadaveric ankle-subtalar joints in quasi-static loading. J. Biomech. Eng. 120, 105-111.

Peden, M., Scurfield, R., Sleet, D., Mohan, D., Hyder, A., Jarawan, E., et al., 2004. World Report on Road Traffic Injury Prevention. The World Health Organization, Geneva. http://www.who.int/violence_injury_prevention/publications/ road_traffic/world_report/en/.

Peldschus, S., Schuller, E., Koenig, J., Gaertner, M., Garcia Ruiz, D., Mansilla, A., 2007. Technical bases for the development of a test standard for impacts of powered two-wheelers on roadside barriers. Proceedings International Technical Conference on the Enhanced Safety of Vehicles .

Pressman, E.K., Cavanaugh, J.L., Woods, J.R., 2002. Physical properties of the chorioamnion throughout gestation. Am. J. Obstet. Gynecol. 187, 672-675.

Quinlan, K.P., Annest, J.L., Myers, B., Ryan, G., Hill, H., 2004. Neck strains and sprains among motor vehicle occupants - United States. Accid. Anal. Prev. 36, 21-27.

Raghupathi, R., Margulies, S.S., 2002. Traumatic axonal injury after closed head injury in the neonatal pig. J. Neurotrauma 19, 843-853.

Robertson, A., Branfoot, T., Barlow, I.F., Giannoudis, P.V., 2002. Spinal injury patterns resulting from car and motorcycle accidents. Spine (PhilaPa 1976) 27, $2825-2830$.

Rudd, R., Crandall, J., Millington, S., Hurwitz, S., Hoglund, N., 2004. Injury tolerance and response of the ankle joint in dynamic dorsiflexion. Stapp Car Crash J. 48, 1-26.

Rupp, J.D., Reed, M.P., Van Ee, C.A., et al., 2002. The tolerance of the human hip to dynamic knee loading. Stapp Car Crash J. 46, 211-228.

Rupp, J.D., Reed, M.P., Jeffreys, T.A., Schneider, L.W., 2003. Effects of hip posture on the frontal impact tolerance of the human hip joint. Stapp Car Crash J. 47, 21-33.

Salzar, R.S., Bass, C.R., Kent, R., et al., 2006. Development of injury criteria for pelvic fracture in frontal crashes. Traffic Inj. Prev. 7, 299-305

Segui-Gomez, M., Lopez-Valdes, F., 2007. Recognizing the importance of injury in other policy forums: the case of motorcycle licensing policy in Spain. Inj. Prev. 6 429-430. 
Siegmund, G.P., Winkelstein, B.A., Ivancic, P.C., Svensson, M.Y., Vasavada, A., 2009. The anatomy and biomechanics of acute and chronic whiplash injury. Traffic Inj. Prev. 10, 101-112.

Smith, D.H., Wolf, J.A., Lusardi, T.A., Lee, V.M., Meaney, D.F., 1999. High tolerance and delayed elastic response of cultured axons to dynamic stretch injury. J. Neurosci. 19, 4263-4269.

Soni, A., Chawla, A., Mukherjee, S., Malhotra, R., 2010. Lateral bending moment threshold of the knee joint - effects of active muscles. Proceedings International Research Council on the Biomechanics of Impact, 211-216 .

Subit, D., Duprey, S., Lau, S., Guillemot, H., Lessley, D., Kent, R., 2010. Response of the human torso to lateral and oblique constant-velocity impacts. Ann. Adv. Automot. Med. 54, 27-40.

Tagliaferri, F., Compagnone, C., Yoganandan, N., Gennarelli, T.A., 2009. Traumatic brain injury after frontal crashes: relationship with body mass index. J. Trauma 66, 727-729.

Takhounts, E.G., Eppinger, R.H., Campbell, J.Q., Tannous, R.E., Power, E.D., Shook, L.S., 2003. On the development of the SIMon finite element head model. Stapp Car Crash J. 47, 107-133.

Takhounts, E.G., Ridella, S.A., Hasija, V., et al., 2008. Investigation of traumatic brain injuries using the next generation of simulated injury monitor (SIMon) finite element head model. Stapp Car Crash J. 52, 1-31.

Ueno, K., Melvin, J.W., Li, L., Lighthall, J.W., 1995. Development of tissue level brain injury criteria by finite element analysis. J. Neurotrauma 12, 695-706.

Uriot, J., Baudrit, P., Potier, P., et al., 2006. Investigations on the belt-to-pelvis interaction in case of submarining. Stapp Car Crash J. 50, 53-73.

Viano, D.C., King, A.I., Melvin, J.W., Weber, K., 1989. Injury biomechanics research: an essential element in the prevention of trauma. J. Biomech. 22, 403-417.
WHO, 2009. Global Status Report on Road Safety: Time for Action. The World Health Organization, Geneva.

Walz, M., 2004. NCAP test improvements with pretensioners and load limiters. Traffic Inj. Prev. 5, 18-25.

Weiss, H.B., Songer, T.J., Fabio, A., 2001. Fetal deaths related to maternal injury. JAMA 286, 1863-1868

Weiss, H.B., 2001. The epidemiology of traumatic injury-related fetal mortality in Pennsylvania, 1995-1997: the role of motor vehicle crashes. Accid. Anal. Prev. 33, 449-454.

Yoganandan, N., Sances, A., Larson, S.J., Weinshel, S.S., Jentzen, J., Pintar, F.A., et al., 1989. Epidemiology and injury biomechanics of motor vehicle related trauma to the human spine. Stapp Car Crash Conference Proceedings 33[892438] .

Yoganandan, N., Pintar, F.A., Kumaresan, S., Boynton, M., 1997. Axial impact biomechanics of the human foot-ankle complex. J. Biomech. Eng. 119, 433-437.

Yoganandan, N., Pintar, F.A., Gennarelli, T.A., Maltese, M.R., Eppinger, R.H., 2001. Mechanisms and factors involved in hip injuries during frontal crashes. Stapp Car Crash J. 45, 437-448.

Zhang, L., Yang, K.H., Dwarampudi, R., et al., 2001a. Recent advances in brain injury research: a new human head model development and validation. Stapp Car Crash J. 45, 369-394.

Zhang, L., Yang, K.H., King, A.I., 2001b. Comparison of brain responses between frontal and lateral impacts by finite element modeling. J. Neurotrauma 18, 21-30.

Zhang, L., Bae, J., Hardy, W.N., et al., 2002. Computational study of the contribution of the vasculature on the dynamic response of the brain. Stapp Car Crash J. 46, 145-164.

Zhang, L., Yang, K.H., King, A.I., 2004. A proposed injury threshold for mild traumatic brain injury. J. Biomech. Eng. 126, 226-236. 